\title{
Genetically-modified insects to combat vector-borne diseases - curtain rise or fall?
}

One of the promising applications of CRISPR-Cas9 in non-humans - the engineering of gene drives to create genetically modified (GM) insects - has garnered significant interest for its potential to control vector-borne diseases. On Dec 17, 2015, the UK House of Lords Science and Technology Committee published a report that emphasizes the woeful inadequacy of the existing national and EU regulatory framework for GM organisms and the need for widespread regulatory reform before this application can reach centre stage. The report calls on the UK Government to launch a field trial of GM insects to test not only the science, but also the regulatory processes and policies. With so many people concerned about the risks of introducing GM organisms into the wild, do the ends justify the means?

More than 1 million lives are lost annually to vectorborne diseases, including an estimated 438,000 lives claimed by malaria alone last year. According to the WHO's most recent estimate, nearly half of the world's population - approximately 3.2 billion people in 97 countries - are at risk of contracting malaria. With the recent emergence and spreading of antimalarial drug resistance, disease eradication remains a primary aim for governments and non-governmental organisations. Efficient vector control could help the WHO achieve its ambitious goal of reducing malaria case incidence and mortality rates by at least $90 \%$ and eliminating the disease in at least 35 countries by 2030 .

It is not difficult to see the appeal of engineering synthetic gene drives for this endeavour, as the Review by Champer, Buchman and Akbari published in this issue of Nature Reviews Genetics makes clear. Synthetic gene drives are based on naturally existing 'selfish' genetic elements, which have developed mechanisms to circumvent Mendelian inheritance rules so as to be passed on to more than the expected $50 \%$ of offspring. By introducing a desirable trait such as pathogen resistance or by targeting a gene for disruption, leading to recessive lethality or sterility, gene drives can be used to rapidly replace or even suppress an entire insect population. Until recently, development of this technology was hampered by substantial technical difficulties, but CRISPR-Cas9 is now surely setting the scene, and major breakthroughs were reported in late 2015 that are likely to speed up the development of techniques to replace or suppress mosquito populations to levels that would not support malaria transmission.

The appropriate regulatory frameworks to accompany these scientific breakthroughs are lacking, however. The Cartagena Protocol on Biosafety currently contains some provisions for its signatory states - which excludes the US - such as measures to restrict the movement of living GM organisms across international boundaries, which is clearly unrealistic for insects. Two existing EU directives that currently underpin the UK's regulations on testing and releasing GM organisms have already proven unfit for purpose in the context of GM crops.

Scepticism remains about the safety of releasing GM organisms that may persist in the wild. Some have even raised concerns about the potential misappropriation of gene drives for biological warfare or for causing economic harm, leading to calls for a moratorium on the research of gene drives and restrictions on the publication of technical information. If the history of GM crops has anything to teach us, it is that full transparency and early engagement with the public will be critical for the acceptance of possible uses of gene drives.

It would be foolish to prematurely dismiss a technology with the potential for such significant public-health, environmental and economic benefits. If the UK is to make the most of its status as a world leader in this area of research, it must effectively stage-manage national and international efforts to ensure that regulatory gaps are filled; that benefits and risks are assessed efficiently; and, most importantly, that the system operates as intended. Commenting on their report, the Earl of Selborne, Chairman of the House of Lords Science and Technology Committee, said: "With a Governmentbacked field trial, an informed public, and regulation that is fit for purpose, and no longer failing lamentably, we will be in a much better position to realise the enormous potential of GM insects." Indeed, a field trial will be essential before gene drives can make their way into the limelight. Ring up the curtain! At least a million lives a year depend on it.

Champer, J., Buchman, A. \& Akbari, O. S. Cheating evolution: engineering gene drives to manipulate the fate of wild populations. Nat. Rev. Genetics http://dx.doi.org/10.1038/nrg.2015.34 (2016). 